Referencia para citar este artículo: Carceller-Maicas, N. (2018). Mundos vitales de palabras: análisis del sufrimiento adolescente mediante narrativas de malestar. Revista Latinoamericana de Ciencias Sociales, Niñez y Juventud, 16(1), 269-283. doi:10.11600/1692715x.16116

\title{
Mundos vitales de palabras: análisis del sufrimiento adolescente mediante narrativas de malestar*
}

\author{
NATÀLIA CARCELLER-MAICAS ${ }^{* *}$ \\ Medical Anthropology Research Center y Universitat Rovira i Virgili, España.
}

\begin{abstract}
Artículo recibido en octubre 31 de 2016; artículo aceptado en enero 16 de 2017 (Eds.)
\end{abstract}
- Resumen (analítico): las elevadas tasas de evitación/infrautilización de los servicios de salud mental adolescente señalan la preferencia de este colectivo por autoatender sus malestares. Integrar los saberes juveniles mediante un enfoque hermenéutico y de interpretación de narrativas permite esclarecer las razones de dicha preferencia pues da a conocer, a partir del análisis de nosologías legas, los modos en los que la juventud percibe, siente y vivencia el malestar. Se empleó metodología cualitativa, realizándose entrevistas semi-estructuradas a una muestra representativa de 105 informantes catalanes y catalanas. Los resultados muestran un continuum nosológico lego que incluye términos coloquiales y biomédicos articulándolos en distintos gradientes de malestar. Normalización y patologización, así como durabilidad y severidad del malestar, devienen conceptos clave en la conformación del continuum. Observándose diferencias por subgrupos de estudio y género.

Palabras clave: adolescencia, juventud, narración, emoción expresada, análisis cualitativo, salud mental, malestares, Cataluña (Tesauro de Ciencias Sociales de la Unesco y Tesauro Descriptores en Ciencias de la Salud).

\section{Vital worlds of words: an analysis of adolescent through emotional distress narratives.}

- Abstract (analytical): Avoiding mental health services indicate that adolescents prefer to engage in self-care to treat their emotional distress. In order to clarify the reasons for this preference the author analyzes the lay nosology that adolescents use to name their emotional distress using a hermeneutic and interpretative approach. The study uses a qualitative methodology through 105 semi-structured interviews with a sample of adolescents and young people from Catalonia (Spain). Results show the existence of a lay nosology continuum, articulating the gradient of emotional distress that forms their vital worlds; temporality, normalization and severity are key concepts that shape this suffering. Differences between subgroups and genders were observed. Recognition of these lay nosologies allows better access to the ways in which emotional distress is perceived, felt and lived.

Este artículo corto en Ciencias Sociales en Antropología Médica se basa en la investigación doctoral de la autora, la cual se inició en Mayo de 2013 y finaliza en 2017. Asimismo la presente investigación se deriva de un estudio de mayor escala "Los malestares de los adolescentes. Estilos de vida, salud mental y estrategias profanas para afrontar la adversidad", en el que la autora fue co-coordinadora técnica e investigadora; el proyecto se inició en Noviembre de 2009 y finalizó en Enero de 2014, y fue financiado por el Ministerio de Ciencia e Innovación del Gobierno de España (CSO2009-08432) y por la Fundació Marató TV3 (090730). La tesis doctoral de la autora titulada: "Por mí mism@ saldré adelante. Percepciones, representaciones y prácticas en torno a los malestares emocionales de adolescentes y jóvenes" le da continuidad al citado proyecto de investigación.

** Doctorado en Antropologia (mención internacional, cum laude). Máster en Antropología Médica y Salud Internacional, otorgado por la Universitat Rovira i Virgili, Tarragona, España. Licenciatura en Antropología Social y Cultural, otorgado por la Universitat Rovira i Virgili, Tarragona, España. Licenciatura en Psicología, otorgado por la Universitat Jaume I, Castelló de la Plana, España. Profesora-consultora en el postgrado de Salud Mental Colectiva de la Universitat Oberta de Catalunya. Investigadora del Medical Anthropology Research Center de la Universitat Rovira i Virgili de Tarragona, España. Profesora del Grado de Antropologia Social y Cultural en la Universidad Nacional de Educación a Distáncia en el centro adscrito Cefad. Orcid: 0000-0002-5774-0558. Índice H5: 4. Correos electrónicos: carcellermaicas@gmail.com, natalia.carceller@urv.cat 
This could lead to improvements in the communication between adolescents and professionals.

Key words: Adolescents, young people, narration, expressed emotion, qualitative analysis, mental health, emotional distress, Catalonia (Social Sciences UnescoThesaurus y Thesaurus Health Sciences Descriptors).

\section{Mundos vitais de palavras: análise do sofrimento adolescente mediante narrativas de mal- estar}

- Resumo (analítico): a evasão dos serviços de saúde mental indica a preferência dos jovens pelo autoatenção de seus desconfortos. Para esclarecer as razões para esta preferência se analisam as nosologias leigas que os jovens usam para nomear aos seus mal-estares a partir de uma aproximação hermenêtica e interpretativa; foram desenvolvidas entrevistas semiestruturadas a uma amostra de adolescentes e jovens na Catalunha (Espanha). Os resultados desvelam a existência de um continuo nosológico que articula os gradientes de mal-estar emocional dos mundos vitais adolescentes sobre aspectos como temporalidade, normalização e severidade. Foram observados diferentes subgrupos de estudo e de gênero. O conhecimento das nosologias leigas permite um acesso aos modos em que o sofrimento é percebido, sentido e vivido e, como consequência, pode implicar numa melhor comunicação entres jovens e profissionais da saúde.

Palavras-chave: adolescencia, juventude, narração, emoções manifestadas, análise qualitativa, saúde mental, mal-estares, Catalunha (Thesaurus de Ciências Sociais da Unesco e Thesaurus Descritores Ciências da Saúde).

-1. Introducción. -2. La importancia de las narrativas. -3. Metodología. -4. Resultados: nosologías legas de malestar emocional. -5. Conclusiones. -Lista de referencias.

\section{Introducción:}

\section{"Los cientificos dicen que estamos hechos de átomos... Pero a mí un pajarito me contó que estamos hechos de historias." Eduardo Galeano}

Los problemas de salud mental son comunes en la adolescencia, especialmente las condiciones clínicas y subclínicas de depresión y ansiedad (Balázs et al., 2013). En Cataluña, la prevalencia de trastornos del humor es del $14,3 \%$ y de los trastornos de ansiedad del $31,9 \%$, pero a pesar de ello la juventud es el grupo de edad que menos utiliza los servicios de salud mental (Merikangas et al., 2010). Diversos estudios señalan que únicamente entre el 18 y el $34 \%$ de adolescentes con trastornos o síntomas de depresión o ansiedad son tratados/ as por profesionales de la salud mental, incluso cuando el servicio se proporciona gratuitamente; este fenómeno es conocido como evitación y/o infrautilización (Alonso et al., 2007; Gulliver, Griffiths, \& Christensen, 2010; Rickwood, Deane, \& Wilson, 2007; Zachrisson, Rödje, \& Mykletun, 2006). En este grupo de edad la infrautilización/evitación de los servicios está asociada, entre otros factores, con la preferencia por buscar formas alternativas de ayuda basadas en estrategias profanas de autoatención (Gulliver et al., 2010; MartínezHernáez, Carceller-Maicas, DiGiacomo, \& Ariste, 2016; Martínez-Hernáez, \& Muñoz, 2010; Martorell-Poveda, Martínez-Hernáez, Carceller-Maicas, \& Correa-Urquiza, 2015). Para entender en profundidad dicha preferencia por la autoatención resulta de especial interés aproximarse a los modelos explicativos de malestar empleados por adolescentes y jóvenes, pues solo así lograremos entender los modos en que éstos/as perciben, sienten y viven el sufrimiento emocional. El análisis de narrativas se considera una estrategia de aproximación clave en esta línea pues permite indagar sobre las vivencias en torno al proceso salud/enfermedad/ 
atención ${ }^{1}$ rescatando los saberes subalternos, los cuales habitualmente quedan silenciados bajo la hegemonía del Modelo Médico Hegemónico $(\mathrm{MMH})$ y su saber dominante, la biomedicina (Menéndez, 1985).

El "malestar emocional" hace referencia a los sufrimientos psíquicos y emocionales emergentes de la condición de ser mujer/ hombre (Burin, \& Velázquez, 1990), "los cuales no implican necesariamente la posesión ni consecución de un diagnóstico psiquiátrico de depresión" (Carceller-Maicas, 2013, p. 299). Remarca el carácter temporal, no definitivo, y por tanto variable, modificable y cambiante de un mal, de algo que produce desazón en la persona; estando alejado de la noción estática característica del diagnóstico patológico psiquiátrico. El malestar emocional es pues un estado temporal circunstancial ligado al mundo afectivo que compila un variado abanico de "padeceres" que pueden estar relacionados con la enfermedad o con percepciones y sentires cercanos a ésta; y abarca desde el más ínfimo y efímero desasosiego hasta los estados depresivos más longevos, formando un contínuum emocional ligado a padecimientos en salud.

Las maneras en las que se expresa el malestar emocional dicen mucho sobre los modos en los que éste es entendido (Cabruja, Íñiguez, \& Vázquez, 2000), así como sobre el modo en que es vivido y afrontado por sus protagonistas. La antropología, gracias a su potencial dialógico y a su posición de escucha activa (MartínezHernáez, 2010) permite dar voz a los actores principales, recopilando, por medio de las narrativas, los saberes legos que de otro modo quedarían invisibilizados tras los discursos hegemónicos (Bruner, 2004). Analizar los discursos profanos en general, y las narrativas de malestar emocional en particular, permite ver los modos en los que se configuran los distintos mundos vitales emocionales; posibilitando evidenciar el rol que el discurso biomédico juega en la concepción de la enfermedad, y en

1 El proceso salud/enfermedad/atención se entiende como un contínuum en el que salud y enfermedad no son dos opuestos cualitativos, sino que entre ambas existe una continuidad (Canguilhem, 1978) en la cual co-existen variedad de matices y posibilidades experienciales. los modos en los que se incorpora en el saber profano, configurando una especie de esqueje a partir del cual se producen nuevas y múltiples formas de concebir la realidad.

Las palabras elegidas para explicar las experiencias de malestar y enfermedad resultan cruciales en la subsecuente experiencia vivida, pues las diferentes maneras de percibir, entender, sentir y vivir el malestar resultan en distintos modos de nombrarlo, y vice-versa; configurando de este modo los "mundos vitales de palabras". Este concepto se inspira en los "mundos morales locales" 2 de Kleinman (2013) pero remarca la importancia de los modelos explicativos y las narrativas sobre las experiencias vividas de malestar en la construcción de estos mundos morales locales.

Debido a que el mundo adolescente/juvenil posee particularidades que lo diferencian del mundo adulto es preciso entenderlo como una entidad cultural en sí misma con su lenguaje y simbología propios. Tal y como diría Geertz: "La cultura de un pueblo es un conjunto de textos. (...) Las sociedades contienen en sí mismas sus propias interpretaciones. Lo único que se necesita es aprender la manera de tener acceso a ellas" (Geertz, 1993, p. 372); resultando las narrativas de malestar juvenil una llave maestra que da acceso a una mejor comprensión de su mundo.

Esta investigación indaga sobre las razones que fundamentan la preferencia de adolescentes y jóvenes por autoatender su malestar emocional (y evitar los servicios de atención profesional), centrándose para ello en conocer y analizar los modelos explicativos que manejan en torno al malestar emocional, analizando asimismo las articulaciones existentes entre el discurso médico hegemónico y los discursos juveniles subalternos; contribuyendo de este modo a la

2 "Los mundos morales locales son escenarios de la experiencia moral que expresan lo que más está en juego para las personas en sus redes locales de relaciones en las comunidades. Estos mundos ya no se limitan a los espacios limitados de la etnografía tradicional, como el pueblo, el lugar de trabajo, la favela o el hogar, sino que se extienden a las redes donde la vida cotidiana se promulga y se realiza, donde la experiencia subjetiva interna está en interacción con las prácticas y compromisos de otras personas. Estos podrían ser transcontinentales y transnacionales, o profesionales y de negocios, así como nacionales. Aquí, los valores vividos del individuo y los de la red pueden estar en sintonía o en conflicto, implicando constantes contestaciones y compromisos" (Kleinman, 2013). 
democratización de los saberes. Este artículo se centra concretamente en la detección y análisis de las nosologías legas ${ }^{3}$ que adolescentes y jóvenes emplean para nombrar su malestar, como primer paso hacia el conocimiento y comprensión de sus modelos explicativos.

\section{La importancia de las narrativas}

Aproximarse al abordaje de los malestares emocionales en adolescentes y jóvenes mediante el análisis de sus narrativas permite "capturar la vívida inmediatez de la experiencia, de la cual el significado no puede separarse" (Beatty, 2013, p. 414). Las narrativas poseen un potencial transformador, pues van más allá del contenido de la narración incluyendo una temporalidad, estableciendo causalidades y produciendo relaciones, lo cual les permite constituirse como vías de acción para el sujeto (Schöngut, \& Pujol, 2015). Es por estos motivos por los que se considera adecuado, tanto a nivel teórico como práctico, abordar el estudio de los malestares emocionales desde el análisis de narrativas. Autores como Beatty mencionan la relación existente entre emoción y narración, $y$ recomiendan el uso de las segundas para lograr el óptimo estudio de las primeras, pues "una gran labor de la antropología será redescubrir la particularidad de la emoción y su dimensión temporal, la cual sólo puede capturarse adecuadamente por medio de la narrativa" (Beatty, 2013, p. 421).

Existe una profunda interrelación entre tres conceptos analíticos clave como son la emoción, la experiencia vivida y la narración, tal y como puede apreciarse en definiciones conceptuales como la propuesta por Shweder para describir el concepto de emoción: "estructuras narrativas complejas que dan forma y significado a experiencias somáticas y afectivas" (Shweder, 1994, p. 37).

3 La Real Academia Española define "nosología" como la: "Parte de la medicina que tiene por objeto describir, diferenciar y clasificar las enfermedades". Inspirado en este término perteneciente al ámbito biomédico el concepto de "nosología lega" refiere, en contrapunto, a la parte del saber popular subalterno que tiene por objeto describir, diferenciar y clasificar los malestares y padeceres vitales; incluyendo por tanto todos aquellos términos, expresiones y nomenclaturas que emplean habitualmente las personas que no están instruidas en el campo de la salud.
Esta investigación aborda los malestares emocionales desde un enfoque hermenéutico en el que las narrativas, los discursos e incluso el lenguaje en sí mismo van más allá del utilitarismo, pues considera que el lenguaje, tal y como lo definen Das y Kleinman, es "una experiencia que permite al sujeto no sólo trasmitir un mensaje, sino que también le permite proyectarse hacia el exterior" (Das, \& Kleinman, 2001, p. 22). Esta proyección del sujeto desde su yo más íntimo y profundo hacia la esfera exterior contextual donde se encuentran los otros es la que posibilita que las personas se aproximen a aquello que Boon llama reflexividad diaria (Boon, 1999), la cual puede ser entendida, en base a las aportaciones del autor, como ese proceso cotidiano en el que las personas casi sin darse cuenta piensan y repiensan continuamente tanto a sí mismas, como a su contexto, y a ellas dentro del contexto. Este aspecto también se evidencia en el proceso de comunicación interpersonal que acontece en el transcurso de la narración, pues tal y como LeVine defiende: "la comunicación interpersonal es el medio a través del cual descubrimos cómo los individuos experimentan sus vidas y cómo las creencias culturales dan forma a dicha experiencia" (LeVine, 1982, p. 293). Sin embargo las creencias culturales no resultan ser las únicas que contribuyen a dar forma a la experiencia, pues si así fuese las experiencias de los miembros de una misma cultura serían todas relatadas e interpretadas de maneras poco más o menos análogas, lo cual dista bastante de la realidad. Los factores sociales juegan igualmente un papel determinante en la percepción y vivencia de la experiencia. Aspectos tales como el género, la edad, los recursos personales, económicos y materiales, así como los roles que se juegan en el contexto tienen un peso determinante en el proceso. Aunque es esencial remarcar que no todo son factores socio-culturales externos, pues existe otra tipología de variables que deviene crucial en lo que a la percepción y la narración de la experiencia de malestar se refiere: el bagaje personal.

El bagaje personal de experiencias previas aporta el toque personal que marca las diferencias en torno al hecho vivido, pues 
ante un mismo acontecimiento o hecho social las distintas personas que lo vivencian lo perciben y reaccionan de distintas formas. Todo este conjunto de factores se encuentra profundamente interrelacionado $\mathrm{y}$ da forma a un complejo entramado que influye en la manera en la que adolescentes y jóvenes viven y narran sus malestares emocionales (Carceller-Maicas, 2013). Es por tanto a partir de las narraciones del malestar vivido y experimentado, y del modo en que éstas son comunicadas, que logramos aproximarnos en mayor medida al sentir encarnado de los actores principales. El uso de las narrativas como vía de aproximación y fuente de información y análisis cualitativa e interpretativa permite, tal y como defiende Amar-Rodríguez "dar a comprender unos hechos que son contados por nuestros informantes con voz propia, a través de la singularidad de su recuerdo, emoción o memoria, sobre la importancia de leer la vida" (Amar-Rodríguez, 2016, p. 978). Narrar ayuda a elaborar la propia vivencia, pero para llegar a narrar en voz alta las vivencias personales y las experiencias vitales significativas a un tercero resulta imprescindible pararse, aunque tan sólo sea por un instante, a rememorarlas. Este rememorar o recordar nunca es neutro ni "objetivo", puesto que se ve influenciado y condicionado por los hechos y circunstancias que configuran la vida de la persona, incluso a pesar de que éstos hayan tenido lugar a posteriori del evento rememorado, pues influyen tanto en la interpretación del hecho recordado como en la emoción que éste causa. Acudir a los orígenes del término da una idea clara y sencilla de este argumento: el término "recordar" proviene del latín re-cordis y significa volver a pasar por el corazón. Cada vez que recordamos reinterpretamos imbuidos por nuestro yo presente en continua e incesante reconstrucción. Tal y como explica Damasio: "Todos nosotros tenemos la experiencia directa de que cuando rememoramos un objeto dado, $o$ cara, o escena, no obtenemos una reproducción exacta sino más bien una interpretación, una versión acabada de reconstruir del original" (Damasio, 1995, p. 153). No existe, por tanto, una objetividad neutra y aséptica en las narrativas, sino que éstas son fruto de la subjetividad de las experiencias y vivencias de la persona que las narra; siendo ésta una de las riquezas que esta técnica nos ofrece, pues "nuestra intención no se centra en exclusividad en estudiar al sujeto sino en conocer su subjetividad" (Amar-Rodríguez, 2016, p. 978). Este recuerdo subjetivo, "condicionado" y cambiante con el transcurso del tiempo y de la vida llevará a narrar el malestar vivido a través de diversos "modelos explicativos". Y es a través de este término (modelos explicativos) propuesto por Kleinman $(1980)^{4}$ que se puede llegar a discernir y evidenciar aquellos aspectos que son de especial importancia para cada individuo en su propia experiencia de malestar; resultando de este modo el análisis de narrativas crucial para lograr vislumbrar dichos aspectos clave.

\section{Metodología}

\section{Diseño de investigación y partici- pantes}

Participaron 105 adolescentes y jóvenes (de entre 17 y 21 años) de todo el territorio catalán seleccionado/as a partir de una muestra representativa de más de tres mil informantes del Panel de Famílies i Infància (PFI), un estudio sociológico longitudinal en cuatro oleadas que inició en 2006 (Institut d'Infància i Món Urbà, 2012; Martínez-Hernáez, Marí-Klose, Julià, Escapa, Marí-Klose, \& DiGiacomo, 2012). La información del PFI versaba sobre aspectos relacionados con estados de ánimo negativos mediante una escala auto-administrada (años 2007 y 2008) y sobre la existencia de un diagnóstico previo de depresión/ansiedad (años 2006 y 2010), además de diversidad de factores sobre consumo de substancias psicoactivas,

$4 \quad$ Modelos Explicativos (EMs): "nociones acerca de un episodio de enfermedad y su posible terapéutica que son empleadas por todos aquellos involucrados en el proceso clínico. La interacción entre los EMs de los pacientes y los EMs de los profesionales es el componente fundamental de la atención en salud. El estudio de los EMs de los profesionales nos remite a cómo ellos entienden y tratan la enfermedad. El estudio de los EMs de los pacientes y familiares expresa cómo ellos dotan de sentido un episodio de aflicción y cómo escogen y valoran los diferentes tratamientos. La investigación de la interacción entre los EMs de profesionales y pacientes nos permite un análisis de los problemas que aparecen en la comunicación clínica" (Kleinman, 1980, p. 105) 
sociabilidad, factores económicos, escolares y familiares.

La selección de la submuestra de 105 informantes que compone la presente investigación se efectuó a través de la técnica propensity matching score, obtenida sobre la base de predictores observables mediante un análisis de regresión logística. Se configuraron tres grupos de participantes: a) Grupo "Diagnóstico": compuesto por informantes con depresión/ansiedad diagnosticada en la $1^{\mathrm{a}}$ o $4^{\mathrm{a}}$ oleada del PFI según el relato de sus progenitores (la pregunta incidía sobre si existía un diagnóstico profesional de depresión/ ansiedad); b) Grupo "Malestar": informantes con malestar auto-percibido de tipo depresivo en las oleadas $2^{\mathrm{a}}$ y $3^{\mathrm{a}}$ pero sin diagnóstico clínico profesional de depresión/ansiedad; y c) Grupo "Control": informantes sin diagnóstico clínico ni malestar emocional auto-percibido. Pese a que la selección inicial fue de 50 jóvenes por subgrupo debido a la pérdida de muestra (por cambio de domicilio, imposibilidad de contacto, o negativa a ser entrevistado/a) finalmente fueron entrevistados/as 105 jóvenes: $37 \mathrm{del}$ Grupo Diagnóstico, 33 del Grupo Malestar, y 35 del Grupo Control, con una proporción de $31 \%$ hombres y $69 \%$ mujeres.

\section{Entrevista:}

Se realizó una entrevista semi-estructurada a los/as 105 informantes. Iniciaba con el relato de una viñeta creada ex profeso en la que se exponía el caso de un/a joven imaginario/a que mostraba síntomas de depresión siguiendo los criterios que establecen manuales al uso como el DSM-IV-TR (American Psychiatric Association, 2002), pero sin explicitar posibles motivos o causas. La entrevista proseguía con una batería de 38 preguntas dirigidas a analizar las narrativas de adolescentes y jóvenes sobre malestares emocionales, las estrategias profanas que emplean para afrontar la adversidad y su visión sobre los dispositivos especializados de atención a la salud mental y los profesionales que en ellos trabajan. La primera pregunta de la entrevista siempre fue ¿Qué le pasa a María/ Juan según tu punto de vista? Continuando con preguntas que iban focalizando progresivamente en la experiencia del/de la entrevistado/a. El/la entrevistador/a no citó ni utilizó previamente la palabra "depresión" o cualquier otra categoría diagnóstica. Se garantizó el anonimato de los/ as participantes durante todo el proceso de investigación, y tanto los/as jóvenes como sus progenitores/as firmaron el consentimiento informado.

El género del personaje de la viñeta inicial fue el mismo que el de la persona entrevistada. Las entrevistas, de una hora y media de duración aproximadamente, fueron grabadas, transcritas, almacenadas, codificadas y analizadas mediante el software cualitativo Atlas.ti. Se realizó inicialmente un análisis temático para identificar los contenidos principales de los datos obtenidos. A continuación se organizó una estructura de codificación de acuerdo con los principios de la teoría fundamentada (Glaser, \& Strauss, 1967), el modelo hermenéutico y el método etnográfico, incluyendo el descubrimiento de tipologías nativas o emic. Posteriormente se revisaron las transcripciones y se aplicaron los códigos incluyendo la identificación y análisis de las excepciones, así como la reflexividad y la revisión entre pares.

El empleo de la viñeta inicial actúa como un "acontecimiento ficcionado" que permite interrumpir el flujo del devenir y condensar el tiempo, poseyendo "las mismas características y cualidades que los acontecimientos reales" (Gómez-Esteban, 2016, p. 142). Estas particularidades de la viñeta posibilitan que los/ as informantes se examinen y se reflexionen sobre acontecimientos imaginados o vividos a través de otros como si ellos/as mismos/as los hubiesen experimentado; permitiéndonos, a partir de dicha ficcionalización, aproximárnos en mayor medida a sus sentires y realidades emocionales.

Viñeta inicial:

María/Juan tiene 17 años y las dos pasadas semanas se ha sentido mal. Se siente triste y vacio/a, y a veces esta irritable. Le cuesta mucho concentrarse. Ya no siente placer por las cosas que siempre le han gustado y no tiene ganas de comer. Le cuesta mucho dormir por la noche y pasa el día sin energía para hacer las actividades que siempre había hecho. 
Las preguntas de la entrevista para indagar en las nosologías legas fueron: 1) ¿Qué le pasa a Maria/Joan según tu punto de vista?; 2) ¿Qué nombres recibe eso que le pasa? ; 3) ¿Me puedes decir qué palabras utiliza la gente de tu edad para hablar de estos estados?

\section{Resultados: nosologías legas de malestar emocional.}

A partir del caso planteado en la viñeta inicial de la entrevista, elaborada incluyendo sintomatologías pertenecientes a la categoría de "Depresión" que expone el DSM-IV-TR, se formularon preguntas sobre el malestar de María/Juan, dirigidas a indagar sobre la nosología lega que emplean adolescentes y jóvenes para referirse al malestar. A partir de dichas cuestiones (preguntas 1, 2 y 3 de la entrevista) los/as informantes dan a conocer su repertorio nosológico, compuesto por más de setenta expresiones y términos diferentes. Las nosologías sugeridas incluyen términos que se posicionan en diversos puntos de un continuum que abarca desde conceptos coloquiales provenientes del argot generacional hasta conceptos formales pertenecientes a los sistemas médicos expertos.

Tabla 1. Nosologías Legas de Malestar.

\begin{tabular}{|c|c|c|}
\hline \multicolumn{3}{|c|}{ TABLA 1: NOSOLOGIAS LEGAS DE MALESTAR EMOCIONAL } \\
\hline Agobiado/a & Está deshecho/a & No tiene ambiciones \\
\hline Amargado/a & Está destrozado/a & Perdido/a por la vida \\
\hline Amparanoiat/Emparanoiado/a & Está en blanco & Pesimismo \\
\hline Angustiado/a & Está en esos días & Pocho/a \\
\hline *Anorexia & Está en la parra & Poco satisfecho/a \\
\hline *Ansiedad & Está en shock & Preocupación \\
\hline Apatía & Está enamorado/a & *Problemas de la adolescencia \\
\hline Autista & Está enfermo/a de algo & Puteado/a \\
\hline Baja autoestima & *Está mal & Que le hagan Bullying \\
\hline Bloqueado/a & Está pocho/a & Raro/a \\
\hline Bulimia & Está tocado/a & *Ratllat/rayado/a \\
\hline Burchado/a & Estresado/a & Se ha dado un mal trago \\
\hline Cabizbajo/a & Frustrado/a & Sin fuerzas \\
\hline Cansado/a & Fuera de sí mismo/a & Sin vida \\
\hline Cerrado/a & Hecho/a mierda & Sufrimiento \\
\hline Confundido/a consigo mismo/a & Hundido/a & *Tiene un bajón/está de bajón \\
\hline Crisis identidad & Indecisión & Tienen una cosa chunga \\
\hline Decaído/a & Inestabilidad & Tontería \\
\hline *Deprimido/a & Infelicidad & *Triste \\
\hline Desanimado/a & Insatisfecho/a & Un colapso \\
\hline Desilusión & Insoportable & Un poco out \\
\hline *Desmotivado/a & Jodido/a & Vacío/a \\
\hline Desorientación & Mustio/a & $*$ Xof/Chof \\
\hline Embobado/a & Nerviosismo & \\
\hline Encaprichado/a & No estás en lo que estás & \\
\hline Esta apagado/a & No se quiere a sí mismo/a & \\
\hline
\end{tabular}


En la Tabla 1 se observan las nosologías mencionadas. Las respuestas se codificaron y agruparon por categorías en base a los tipos de malestar, resultando a partir de esta codificación setenta y cinco nosologías distintas. De éstas setenta y cinco sólo cinco de ellas (las resaltadas en la Tabla 1 en negrita) provienen del ámbito médico clínico, perteneciendo el resto al ámbito de lo coloquial y cotidiano. Al analizar los resultados atendiendo a la frecuencia con la que las expresiones son mencionadas se observa un hecho interesante: de los diez términos más empleados (marcados con asterisco en la Tabla 1), tres pertenecen al ámbito clínico (depresión, ansiedad y anorexia), siendo la "Depresión" la categoría que ocupa la primera posición, desmarcándose ampliamente del resto de terminologías.

Existen matices relevantes que muestran las diferencias existentes entre unas y otras nosologías, lo cual puede apreciarse a partir del siguiente análisis de algunas de las más destacadas. Tal y como explican los/as adolescentes y jóvenes para ellos/as "Estar chof/xof" significa "estar diferente", "bajo moralmente". Esta expresión onomatopéyica se utiliza para referir aquellas situaciones: "cuando te sientas, dejas ir un suspiro, o sea que descansas, que estás como apagado, como caído ¿sabes? No tienes ganas de hacer nada, estás cansado de todo" (177_HombreControl) y surge inevitablemente un "buff" (184 HombreControl), la usan cuando están "sin ganas de nada" y "en plan triste", cuando "estás como que no existe nada a tu alrededor, que es solo tu mundo y de ahí no sales" (3 MujerDiagnóstico). Frecuentemente se asocia "estar chof/xof" con "tener/estar de bajón" y "estar rayado/a”, otorgándoseles de este modo posiciones equivalentes dentro del continuum de gravedad del malestar. No obstante, aunque las tres expresiones aluden a niveles similares de malestar, principalmente en base a su carácter de escasa temporalidad: "puedes estar así dos dias y luego te animas (...) es temporal” (81_ MujerMalestar); "estar rayado/a" presenta un matiz diferencial con respecto las otras dos, pues a pesar de que "Chofy rayada vienen a ser igual, rayada es más por algo concreto, pero luego te pasa" (4_MujerDiagnóstico).

La nosología considerada más preocupante por nuestros/as informantes es la "Depresión" pues hace referencia, según ellos/as mismos/ as explican, a estados más graves y severos de malestar. Al inicio de la entrevista, tras la lectura de la viñeta, a muchos/as les vino a la mente el término depresión pero no lo consideraron adecuado para describir lo sucedido en ella porque dijeron que lo veían un término demasiado fuerte. En base a sus modelos explicativos adolescentes y jóvenes interpretan el suceso de la viñeta de modo distinto a como lo entiende el sistema biomédico, pues tal y como se aprecia en el discurso de nuestras/os informantes éstos/as tienden a despatologizar la vivencia y llevarla más hacia el ámbito de lo cotidiano:

“¿Depresión? Bueno, depresión yo lo entiendo más como algo que se tiene que tratar médicamente, yo... a lo mejor es un inicio, pero las personas con depresión... yo he visto personas con depresión y muestran sintomas que diría, mucho más graves. Yo no lo llamaría a esto "depresión", pero bueno, siempre puede ser un principio" (49_MujerMalestar).

Resulta interesante cómo a pesar de ser la nosología más frecuentemente citada es considerada la que indica mayor gravedad, aun cuando muchos/as jóvenes no consideran que el caso de la viñeta necesite de atención médica especializada, pues opinan que se corresponde con una situación cotidiana. Este hecho que a primera vista puede resultar paradójico esconde un matiz interesante que da explicación a este contrasentido. Es a partir del análisis del conjunto de narrativas que hayamos la respuesta a este hecho. La nosología "Depresión" es empleada por la juventud para nombrar dos situaciones completamente distintas en cuanto a la severidad del malestar se refiere, pudiéndola encontrar en dos puntos diametralmente distintos del continuum de gravedad del malestar. Por un lado indica el grado más elevado de malestar, catalogándola como una enfermedad que es necesario abordar y tratar con profesionales sanitarios especializados, considerando que la 
gravedad de lo sucedido ha de ser extrema para producir depresión: "[Es] como una especie de depresión pero que no se puede elevar al grado de depresión porque, evidentemente con 17 años tiene que ser algo muy grave para tener una depresión" (154_MujerControl).

Pero por otro lado esta nosología, originariamente nacida dentro del ámbito clínico, es también empleada por los/as jóvenes para referir un estado de malestar de poca relevancia que queda dentro de la normalidad cotidiana: "Está deprimido, pero exagerando un poco la situación. No está deprimido del todo en el tema médico, pero se siente mal, está triste, está decaído" (168_MujerControl). La "depresión" se configura así en este caso como un término coloquial que ocupa una posición leve dentro del continuum; distanciándose de su similitud con un estado de enfermedad.

Estos dos contextos tan dispares pero a la vez considerados igualmente adecuados por los/as jóvenes para emplear el citado término explican la elevada frecuencia de respuesta que muestra esta nosología. La "depresión" resulta pues una nosología clave en el estudio de los malestares emocionales en jóvenes, puesto que se configura como un concepto-bisagra que refiere tanto a los estados más severos como a los más leves y normalizados.

"P. ¿Qué es depresión para ti?

R. Pues no sé, sabrán los médicos, creo que es algo del cerebro y eso, pero que se dice a estados de ánimo, a veces "depresión" y a lo mejor no lo es. No sé, [sería] como que "está rayado", o que está así, "decaído", "desanimado" (149_MujerControl).

La clave de dicha ambivalencia reside no sólo en el término en sí mismo, sino también en el modo de expresarlo. Los estados leves responden frecuentemente a "estar depre": "Oye estoy mal, estoy depre y no tengo ganas de comer" (76_MujerMalestar); en cambio los estados severos suelen referirse como "tener depresión". El análisis etimológico de las expresiones empleadas da cuenta de las diferencias entre los estados a los que ambas aluden. En "estar depre" se emplea el verbo "estar", que connota momentaneidad, brevedad, y hace referencia a un estado pasajero; además el hecho de abreviar el término "depresión" y dejarlo sólo en "depre" resulta también especialmente significativo. Al parecer del mismo modo que se reduce el término se reduce con él la carga dramática que posee. Se reduce su gravedad y la cantidad de malestar que conlleva. Da la impresión de que al eliminarle letras se le eliminan también los pesares que porta, aligerando así al mismo tiempo la carga del término y la del malestar al que se refiere. La expresión "tener depresión" en cambio emplea el verbo "tener" el cual implica posesión y mantenimiento, denotando un estado de mayor duración en el tiempo ${ }^{5}$. Alude así a una característica definitoria e intrínseca del individuo y connota perdurabilidad, correspondiéndose de este modo a las características comúnmente atribuidas a las etiquetas clínicas diagnósticas presentes en el DSM. Esta nosología puede agravarse aún más cuando en lugar de los verbos "estar" y "tener" se emplea el verbo "ser"; pues si "tener" ya implica una perdurabilidad que incrementa la gravedad de la experiencia de malestar, "ser" hace que la depresión pase a incorporarse (embodied) como una característica intrínseca, definitoria e inmanente de la persona.

El término "depresión" ejerce de hilo conductor a través del cual se da sentido, significado y orden al resto de nosologías inscritas en el continuum del malestar emocional; pues a partir de la explicación del significado que el término "depresión" tiene para los/as jóvenes se empieza a desenmarañar la trama y surgen a la luz las particularidades por medio de las comparaciones entre las distintas nosologías, construyendo un gradiente nosológico dentro del continuum del malestar emocional.

El análisis muestra que la temporalidad del malestar deviene un factor clave a la hora de considerar la gravedad del mismo. Los padeceres más momentáneos son considerados de carácter más leve, en cambio, a medida que el malestar se extiende en el tiempo y su duración pasa de un momento a unos días, y de unos días a una temporada, la gravedad se incrementa, y lo que podía considerarse normal

RAE. Ver acepciones 2 y 3 :

http:/lema.rae.es/drae/srv/search?id=48WFY8XXaDXX2R82DbOq 
y típico de la adolescencia pasa a aproximarse al polo de lo patológico y a entenderse como una enfermedad.

"[La depresión] es un estado más crítico, más acentuado [que estar triste]. Una depresión es bastante más. Puedes estar triste, puedes tener unos días que estás mal, y luego estar bien. La depresión [en cambio] es que estás en un estado continuo de tristeza..." (12 MujerDiagnóstico).

"Estar triste"... esto, bueno, todo es momentáneo, ¿no?, pero la tristeza supongo que es más momentánea que la “depresión”, que ya llevas un periodo" (180_HombreControl).

"P: ¿Qué diferencia habrá entre ese momento y la depresión en sí?

R: Que la depresión es una enfermedad que tiene un largo período de tiempo y que tienes que tratarte con psicólogo y medicación" (147_MujerControl).

La temporalidad dē malestar se haya, según los/as jóvenes, profunda e íntimamente vinculada a la gravedad del mismo. Tal y como defiende Amador-Baquiro "las temporalidades sociales son recursos mentales (reguladores) que permiten a los seres humanos y grupos sintetizar, integrar y coordinar el mundo social" (Amador-Baquiro, 2016, p. 1327), jugando pues un papel fundamental en la manera en la que los/as jóvenes conciben el malestar emocional. Los resultados muestran que cuanto más tiempo dura el malestar mayor es su gravedad. "Estar de/tener un bajón", "estar rayado", "estar de mal rollo" o "estar triste" son estados breves, momentáneos, pasajeros, considerados por los/ as jóvenes como parte normal de su día a día. Se observa pues, llegados a este punto, cómo la normalización de las vivencias de malestar juega también un papel relevante en las nosologías empleadas y en los significados que poseen. Aquellas situaciones que quedan dentro de la normalidad y cotidianeidad configuran lo que ellos/as mismos/as categorizan como "estar normalmente deprimido". El "estar normalmente deprimido" o el "being normally depressed" es para ellos/as una consecuencia de las situaciones de la vida cotidiana e incluye situaciones habituales como tener problemas en el instituto, tener problemas con los familiares y/o amigos, e incluso estar enamorado (Martínez-Hernáez, Digiacomo, CarcellerMaicas, Correa-Urquiza, \& Martorell-Poveda, 2014). Debido a esta cotidianeidad la solución a esta "depre normalizada" ha de ser encontrada dentro del contexto de la vida cotidiana y de las relaciones sociales, haciéndole frente mediante estrategias de auto-atención (Martorell-Poveda et al., 2015), pues para los/as adolescentes este "estar normalmente deprimido" no es un estado inusual ni patológico, sino frecuente y normalizado.

El análisis de narrativas muestra que cuando se habla de malestares de tipo depresivo existe una relación inversamente proporcional entre la temporalidad y la normalización del malestar, pues cuanto más perdura el malestar más se aleja del ámbito de lo normal para ir aproximándose progresivamente al polo de lo patológico. Llegados a este punto del continuum en el que hallamos "tener depresión" o "ser depresivo" como las nosologías que denotan mayor severidad, la normalización de la experiencia de malestar desaparece y pasa a ser considerada una enfermedad; quedando en este caso su abordaje y solución dentro del ámbito médico especializado, debiendo ser tratada por profesionales de la salud mental.

Por último es necesario señalar que al analizar los resultados atendiendo a los subgrupos y al género de los/as informantes se evidencian diferencias en la frecuencia de las nosologías más empleadas (ver tabla 2). Pese a que la "Depresión" es la nosología más señalada en todos los subgrupos, es el grupo "Diagnóstico" quien más veces la reporta, seguido por el grupo "Malestar" y por último por el grupo "Control". Además al disgregar los datos por género se observa que son las mujeres las que lo destacan con mayor frecuencia, tanto en la muestra general como en cada grupo en particular. La segunda nosología más nombrada, "tristeza", no muestra diferencias destacadas en este aspecto; en cambio sí que se observan en la tercera, "rallado/a", en la que es el grupo "Control" quien la señala con mayor frecuencia, especialmente las mujeres. Este patrón diferencial de respuestas resulta interesante, pues son las personas más familiarizadas con 
los dispositivos de atención (las que han sido diagnosticadas, Grupo Diagnóstico) las que más aluden a la depresión como nosología explicativa del malestar, en cambio, aquellas más alejadas de los dispositivos, las pertenecientes al grupo control, son quienes más destacan una de las nosologías más normalizadoras y coloquiales del continuum, "rallado/a" (ver tabla 2).

Tabla 2. Nosologías legas de malestar más frecuentes distribuidas por subgrupos y género.

\begin{tabular}{|c|c|c|c|c|c|c|c|c|c|c|c|c|}
\hline \multirow[b]{3}{*}{$\begin{array}{l}\text { Nosologías más } \\
\text { referidas: }\end{array}$} & \multicolumn{9}{|c|}{ Grupo } & & & \\
\hline & \multicolumn{3}{|c|}{ Diagnóstico } & \multicolumn{3}{|c|}{ Malestar } & \multicolumn{3}{|c|}{ Control } & \multicolumn{3}{|c|}{ Total } \\
\hline & $\mathrm{H}$ & $\mathrm{M}$ & $\mathrm{T}$ & $\mathrm{H}$ & M & $\mathrm{T}$ & $\mathrm{H}$ & M & $\mathrm{T}$ & $\mathrm{H}$ & M & $\mathrm{T}$ \\
\hline Depresión & 12 & 20 & 32 & 8 & 18 & 26 & 6 & 16 & 22 & 26 & 54 & 80 \\
\hline Triste & 3 & 5 & 8 & 3 & 7 & 10 & 4 & 7 & 11 & 10 & 19 & 29 \\
\hline Rallado/a & 4 & 4 & 8 & 2 & 5 & 7 & 1 & 12 & 13 & 7 & 21 & 28 \\
\hline Xof/Chof & 1 & 4 & 5 & 0 & 4 & 4 & 2 & 2 & 4 & 3 & 10 & 13 \\
\hline Tiene bajón & 2 & 1 & 3 & 2 & 2 & 4 & 1 & 3 & 4 & 5 & 6 & 11 \\
\hline $\begin{array}{l}\text { N Total respuestas de } \\
\text { las } 75 \text { nosologías }\end{array}$ & 43 & 61 & 104 & 31 & 61 & 92 & 27 & 71 & 98 & 101 & 193 & 294 \\
\hline \multicolumn{13}{|c|}{ H:Hombre; M: Mujer; T: Total } \\
\hline
\end{tabular}

\section{Conclusiones}

El análisis de narrativas de malestar como vía de aproximación y abordaje de los malestares emocionales en adolescentes y jóvenes es una herramienta de investigación que permite acceder a los modos en que dan forma a sus padecimientos (Del Monaco; 2015), así como cuestionar la realidad y desvelar los múltiples significados intersubjetivos de lo vivido (GarcíaHuidobro, 2016). Esta estrategia investigativa reivindica la democratización de los saberes al poner en valor los discursos legos y subalternos al tener en cuenta las múltiples producciones de subjetividad inmersas en los relatos (Güelman, \& Borda, 2014). Es a partir de este "dar voz" a adolescentes y jóvenes, y a poner en valor sus conocimientos sobre sus propias vivencias, que se evidencia la existencia de un modelo explicativo del malestar emocional distinto al del saber biomédico. Tanto la preferencia de adolescentes y jóvenes por autoatender su malestar emocional como las elevadas tasas de infrautilización/evitación de los servicios profesionales por parte de este colectivo hallan su explicación en las diferencias existentes entre ambos modelos explicativos (lego y experto); pues tal y como evidencia el análisis de narrativas realizado aquello que para unos/ as (profesionales) pertenece al ámbito de lo patológico para los/as otros/as (adolescentes y jóvenes) queda dentro del ámbito de la normalidad, siendo por tanto innecesario (a ojos de los/as adolescentes y jóvenes) recurrir a los servicios profesionales para solventarlo. El amalgama de nosologías legas surgido del análisis de narrativas ha permitido en primer lugar aproximarse en detalle a las maneras en las que la juventud percibe, interpreta, siente y experimenta el malestar emocional; posibilitando a posteriori realizar comparativas con las nosologías profesionales clínicas, detectando a partir de éstas las semejanzas, diferencias y articulaciones que tienen lugar entre ambos modelos explicativos. Esto último se hace más fácilmente observable gracias a la estructuración de las nosologías legas juveniles en un continuum nosológico propio, el cual abarca desde términos coloquiales (rallado/a, xof, etc.) que aluden a malestares leves, breves, cotidianos y normalizados, hasta conceptos que han permeado desde el sistema médico experto (depresión, ansiedad, etc.), los cuales refieren malestares más severos, duraderos, insólitos y patológicos. 
Así pues podemos afirmar que el análisis de narrativas ha permitido evidenciar: las especificidades y particularidades de los distintos gradientes nosológicos del malestar, el papel clave que la nosología "depresión" desempeña en la construcción del modelo juvenil al actuar como concepto-bisagra que hace de hilo conductor en la configuración del continuum nosológico de malestar, y el papel esencial que la temporalidad y la normalización juegan en el modelo explicativo juvenil del malestar emocional. Asimismo las diferentes frecuencias de respuesta en las nosologías más destacadas observadas a partir del análisis por subgrupos y género (Tabla 2) podrían estar señalando el modo en que el discurso biomédico permea en los modelos explicativos legos de la juventud sobre el malestar emocional; pues da la impresión de que cuanto mayor (más frecuente) es el contacto y experiencia de la juventud con el sistema biomédico mayor es la influencia que éste ejerce sobre los modelos explicativos legos. No obstante, para poder validar esta hipótesis surgida en el proceso de investigación es necesario indagar en profundidad sobre la frecuencia, intensidad y especificidades de las experiencias y contactos que los/as jóvenes han tenido (y tienen) con los sistemas de atención a lo largo de sus vidas, resultando por tanto este punto una de las limitaciones de la presente investigación. Para atajar dicha limitación se propone como investigación futura realizar una nueva oleada que incremente el alcance longitudinal del estudio en la cual se indague en profundidad sobre: 1-la existencia (o no) de experiencias con los servicios profesionales; 2-la frecuencia, calidad y particularidades concretas de la atención recibida; 3-la (in)satisfacción con el servicio y la atención recibida; 4-así como la opinión que tiene la juventud sobre los dispositivos existentes y cómo ha ido variando ésta con el paso de los años y de las experiencias vividas; empleando para ello nuevas entrevistas y cuestionarios, y realizando historias de vida a informantes clave.

En conclusión podemos afirmar que los resultados obtenidos en la presente investigación nos permiten disponer de nueva información sobre los modelos explicativos adolescentes/

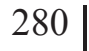

juveniles, la cual nos permite acercarnos a una mayor compresión de su realidad y de sus mundos vitales de palabras; pues nos muestra las similitudes y discrepancias existentes entre los modelos explicativos lego y experto, los modos mediante los que el discurso médico se impregna en los discursos legos, y las maneras en que adolescentes y jóvenes de-construyen y reinterpretan dicha terminología experta y la incorporan en sus propios modelos explicativos del malestar (agency). La construcción de las narrativas de aflicción se configura así como una toma de agencia al auto-constituirse como alternativas a las (meta)narrativas dominantes (Baglia, \& Bonet-Martí, 2009), incorporando una visión del malestar basada en experiencias subjetivas e intersubjetivas encarnadas, y no en la evidencia biomédica. Esta comprensión del malestar desde la visión adolescente muestra un modelo explicativo repleto de expresiones y categorías nosológicas propias que hablan del modo en el que la juventud percibe, siente $\mathrm{y}$ vive el malestar. Conocer y comprender estos modelos explicativos juveniles con todas sus articulaciones y complejidades permite entender mejor sus sentires y padeceres, y los medios por medio de los cuales se configuran y afianzan en sus modos de ser y estar en el mundo. Estudios como el de Bunge, Maglio, Carrea y Entenberg, entre otros ${ }^{6}$, muestran que para adolescentes y jóvenes "poder expresarse" en su relación con los/as profesionales de la salud mental es un aspecto clave en la relación terapéutica, valorándolo incluso como más positivo que los progresos obtenidos (Barber, Tischler, \& Healy, 2006; Biering, 2010; Bunge, Maglio, Carrea, \& Entenberg, 2016); siendo clave que dicha expresión puedan realizarla empleando sus propias palabras y expresiones, en definitiva, sus propios modelos explicativos. Este conocimiento del mundo juvenil puede contribuir a mejorar las relaciones entre adultos/as (profesionales y/o familiares) y jóvenes al posibilitar la existencia de un diálogo comprensivo entre ellos/as, y un acercamiento a

6 Estudios anteriores como el de Barber et al. (2006) y el de Biering (2010) hallaron resultados similares, mostrando que las habilidades comunicacionales en la relación profesionalpaciente son un aspecto crucial cuando se trata de adolescentes.

Rev.latinoam.cienc.soc.niñez juv 16 (1): 269-283, 2018 http://revistalatinoamericanaumanizales.cinde.org.co doi:10.11600/1692715x.16116 
su mundo vital que permita el acompañamiento de adolescentes y jóvenes en su proceso de crecimiento contribuyendo de manera asertiva y facilitadora en la gestión de los malestares emocionales en esta etapa vital.

\section{Lista de referencias}

Alonso, J., Codony, M., Kovess, V., Angermeyer, M. C., Katz, S. J., Haro, J. M., ... Brugha, T. S. (2007). Population level of unmet need for mental healthcare in Europe. $\mathrm{Br} J$ Psychiatry, 190, 299-306.

Amador-Baquiro, J. C. (2016). Jóvenes, temporalidades y narrativas visuales en el conflicto armado colombiano. Revista Latinoamericana de Ciencias Sociales, Niñez y Juventud, 14(2), 1313-1329. doi:1 0.11600/1692715x.14229080915

Amar-Rodríguez, V. (2016). Leer la vida. Una investigación desde la perspectiva narrativa. Revista Latinoamericana de Ciencias Sociales, Niñez y Juventud, 14(2), 975-986. doi:10.11600/169271 5x.14206261015

American Psychiatric Association (APA). (2002). Manual Diagnóstico y Estadístico de los Trastornos Mentales DSM-IV-TR. Barcelona: Masson.

Baglia, B., \& Bonet-Martí, J. (2009). La construcción de narrativas como método de investigación psicosocial. Prácticas de escritura compartida. Forum: Qualitative Social Research, 10(1), Art. 8.

Balázs, J., Miklósi, M., Keresztény, A., Hoven, C. W., Carli, V., Wasserman, C. ... Wasserman, D. (2013). Adolescent subthreshold-depression and anxiety: Psychopathology, functional impairment and increased suicide risk. J Child Psychol Psychiatry, 54(6), 670-677.

Barber, A. J., Tischler, V. A., \& Healy, E. (2006). Consumer satisfaction and child behaviour problems in child and adolescent mental health services. Children Health Care, 10(1), 9-21.

Beatty, A. (2013). Current emotion research in anthropology: Reporting the field. Emotion Review, 5(4), 414-422. doi:10.1177/1754073913490045

Biering, P. (2010). Child and adolescent experience of satisfaction with psychiatric care: A critical review of the research literature. Journal of Psychiatric and Mental Health Nursing, 17(1), 65-72.

Boon, J. A. (1999). Verging on extra-vagance: Anthropology, history, religion, literature, arts... showbiz. Princeton: Princeton University Press.

Bruner, J. (2004). Life as narrative. Originally published. Social Research, 71(3), 691710.

Bunge, E. L., Maglio, A. L., Carrea, G., \& Entenberg, G. (2016). Satisfacción y tipo de servicios de salud mental para niños y adolescentes. Revista Latinoamericana de Ciencias Sociales, Niñez y Juventud, 14(1), 401-415. Doi:10.11600/169271 5x.14127250615

Burin, M., \& Velázquez, S. (1990). El Malestar de las mujeres: la tranquilidad receptada. Buenos Aires: Paidós.

Cabruja, T., Íñiguez, L., \& Vázquez, F. (2000). Cómo construimos el mundo: relativismo, espacios de relación y narratividad. Anàlisi, $25,61-94$.

Canguilhem, G. (1978). Lo normal y lo patológico. México, D. F.: Siglo XXI.

Carceller-Maicas, N. (2013). Los senderos de las emociones. En A. Martínez-Hernáez, S. Digiacomo, \& L. Masana(Eds.), Evidencias y narrativas en la atención sanitaria. Una perspectiva antropológica (pp. 297-322). Tarragona: Publicacions URV, Associação Brasileira da Rede Unida.

Damasio, A. (1995). El error de Descartes. La emoción, la razón y el cerebro humano. Barcelona: Destino.

Das, V., \& Kleinman, A. (2001). Introduction. En V. Das, A. Kleinman, M. Lock, M. Rampele, \& P. Reynolds, (Eds.), Remaking a world: Violence, social suffering, and recovery (pp. 1-30). Berkeley: University of California Press.

Del Monaco, R. (2015). La experiencia de padecer migraña: el análisis de narrativas como estrategia metodológica para el estudio de este dolor crónico. Revista 
Latinoamericana de Metodología de la Investigación Social, 9(5), 22-35.

García-Huidobro, R. (2016). La narrativa como método desencadenante y producción teórica en la investigación cualitativa. Empiria. Revista de Metodología de Ciencias Sociales, 34, 155-178. doi:34.2016.16526

Geertz, C. (1993 [1973]). La interpretación de las culturas. Barcelona: Gedisa.

Glaser, B., \& Strauss, A. (1967). The discovery of grounded theory: Strategies for qualitative research. New York: Aldine Publishing Company.

Gómez-Esteban, J. H. (2016). El acontecimiento como categoría metodológica de investigación social. Revista Latinoamericana de Ciencias Sociales, Niñez y Juventud, 14(1), 133-144. doi:10. 11600/1692715x.1418110815

Güelman, M., \& Borda, P. (2014). Narrativas y reflexividad: los efectos biográficos del enfoque biográfico. Revista Latinoamericana de Metodología de las Ciencias Sociales, 4(1), 1-16.

Gulliver, A., Griffiths, K. M., \& Christensen, H. (2010). Perceived barriers and facilitators to mental health helpseeking in young people: A systematic review. BMC Psychiatry, 10, 113-123.

Institut d' Infància i Mon Urbà. (2012). Panel de Familias e Infancia. Barcelona: Institut d' Infància i Mon Urbà. Recuperado de: http://www.ciimu.org/ima- ges/stories/ CIIMU/Estudios e Investigaciones/ 2panel-fam/14presentacio cggranell.pdf

Kleinman, A. (1980). Patients and healers in the context of culture: An exploration of the borderland between anthropology, medicine, and psychiatry. Los Ángeles: University of California Press.

Kleinman, A. (2013). Why theory matters three more social theories: Social suffering, biopower, and local moral worlds a critical sociology of global health III. Harvard: Harvard University.

LeVine, R. A. (1982). Culture, behaviour and personality. New York: Aldine.

Martínez-Hernáez, A. (2010). Dialógica, etnografia e educação em saúde. Rev. Saúde
Pública, 44(3), 399-405. doi:10.1590/ S0034-89102010005000016

Martínez-Hernáez, A., Digiacomo, S., Carceller-Maicas, N., Correa-Urquiza, M., \& Martorell-Poveda, M. A. (2014). Nonprofessional-help-seeking among young people with depression: A qualitative study. BMC Psychiatry, 14(1), 124-133. doi:10.1186/1471-244X-14-124.

Martínez-Hernáez, A., Carceller-Maicas, N., DiGiacomo, S. M., \& Ariste, S. (2016). Social support and gender differences in coping with depression among emerging adults: A mixed-methods study. Child and Adolescent Psychiatry and Mental Health, 10, 2-13.

Martínez-Hernáez, A., Marí-Klose, M., Julià, A., Escapa, S., Marí-Klose, P., \& DiGiacomo, S. (2012). Adolescent daily smoking, negative mood-states and the role of family communication. Gaceta Sanitaria, 26, 421-428. doi:10.1016/j. gaceta.2011.09.030

Martínez-Hernáez, A., \& Muñoz, A. (2010). Un infinito que no acaba. Modelos explicativos sobre la depresión y el malestar emocional entre los adolescentes barceloneses. Salud Mental, 33(2), 145-152.

Martorell-Poveda, M. A., Martínez-Hernáez, A., Carceller-Maicas, N., \& CorreaUrquiza, M. (2015). Self-care strategies for emotional distress among young adults in Catalonia: A qualitative study. International Journal of Mental Health Systems, 10(9), 9-21. doi:10.1186/s13033015-0001-2

Menéndez, E. L. (1985) Modelo Médico Hegemónico, crisis socioeconómica y estrategias de acción del sector salud. Cuadernos Médicos Sociales, 33, 3-34.

Merikangas, K. R., He, J. P., Burstein, M., Swanson, S. A., Avenevoli, S., Cui, L., ... Swendsen, J. (2010). Lifetime prevalence of mental disorders in U. S. adolescents: Results from the national comorbidity survey replication. $J$ Am Acad Child Adolesc Psychiatry, 49(Suppl.), 980-989.

Rickwood, D., Deane, F., \& Wilson, C. (2007). When and how do young people 
seek professional help for mental health problems? Med J Aust, 187(Suppl 7), S35-S39.

Schöngut, N., \& Pujol, J. (2015). Relatos metodológicos: difractando experiencias narrativas de investigación. Forum Qualitative Sozialforschung/Forum: Qualitative Social Research, 16(2), Art. 24. Recuperado de: http://nbn-resolving. de/urn:nbn:de:0114-fqs1502243.

Shweder, R. A. (1994). 'You're not sick, you're just in love': Emotion as an interpretive system. En P. Ekman, \& R. J. Davidson (Eds.), The nature of emotion: Fundamental questions (pp. 23-44). Oxford: University Press.

Zachrisson, H. D., Rödje, K., \& Mykletun, A. (2006). Utilization of health services in relation to mental health problems in adolescents: A population based survey. BMC Public Health, 16, 6-34. 